\section{Around the Solar system in 1,800 days}

Following the complete success of Pioneer 10, NASA has decided to retarget Pioneer 11, now en route for Jupiter, rather than simply to duplicate the earlier mission. As a result, Pioneer 11 will now swing by Jupiter, using that planet's gravity to assist it into a new orbit and a rendezvous with Saturn late in 1979. And that will pave the way for the planned Mariner missions to Jupiter and Saturn, which include the possibility of putting at least one of the Mariner craft in orbit about Saturn.

Pioneer 11 has now passed the asteroid belt and is more than 4.5 astronomical units (AU) from the Earth; its new path should take it to an encounter with Jupiter on December 2 or 3 this year. The latest Pioneer will pass much closer to the cloud tops of Jupiter than its predecessor $(27,000$ miles as against 81,000 miles, according to a report in Aviation Week \& Space Technology, 100, 18; March 25, 1974). This close approach is essential if good use is to be made of the slingshot effect of Jupiter's gravity and using such approach can only be contemplated in the light of the Pioneer 10 data. Those data showed the structure of magnetosphere and radiation belts sufficiently clearly that the Pioneer project scientists are confident that Pioneer 11 's new path will take it through a gap in those belts, so that in spite of its close approach to Jupiter it will receive less damaging radiation (by some $75 \%$ ) than did Pioneer 10.

But the actual path followed by Pioneer 11 after the Jupiter encounter is almost as interesting as the objective itself. Like the recent Mariner 10 Venus / Mercury mission, which was the first to utilise a gravity-assisted trajectory, Pioneer 11 will actually be slowed down by its encounter with the first planet it passes (Jupiter). The new path will take the Pioneer across the leading edge of the planet (Pioneer 10 passed the trailing edge and was accelerated above escape velocity for the Solar System) and in a loop back across the Solar System, to meet Saturn, eventually, on the opposite side of the Sun from the Jupiter encounter.

The slow journey back across the Solar System will take nearly five years, with Saturn being reached some time in October 1979. The closest approach to the Sun will be some $3.5 \mathrm{AU}$ but the space probe will not have to face the hazard of the asteroid belt again, since its new path will take it out of the ecliptic plane. Clearly, this will provide a wealth of information about interplanetary space. But what are the prospects for the Saturn encounter itself?

Saturn orbits some 9.5 AU from the Sun, at which distance radio signals take $1.5 \mathrm{~h}$ to reach Earth. It would be asking too much to expect spectacular information to be relayed by the first probe to visit Saturn-but as long as the radio transmitter is still functioning useful data could be obtained simply by flying Pioneer 11 through the rings of Saturn to see if it hits anything. That would at least indicate the limits of safe orbits for the following Mariner craft.

It looks as if that at least should be possible, and some scientific information may also be gleaned as a bonus. The spacecraft's nuclear isotope generators were designed to produce 100 W five years after launch (April 1978 in the case of Pioneer 11) but judging from the experience gained by Pioneer 10 the generators are likely to last rather longer than anticipated. It seems feasible that all the experiments could still be operating in October 1979, with sufficient power available to relay their findings, and perhaps even pictures, over Pioneer 11's 10-W transmitter.

Footnote: It seems that the Sunday Mirror has even more faith in the versatility of NASA spacecraft and the power of the gravity-assist technique than NASA project scientists themselves. In the March 31 edition of that newspaper we were presented with a Mariner 10 picture of Mercury with a caption which ended "Mariner 10 is now hurtling through space ready to take pictures of Saturn, the ringed planet . . . in 1979".

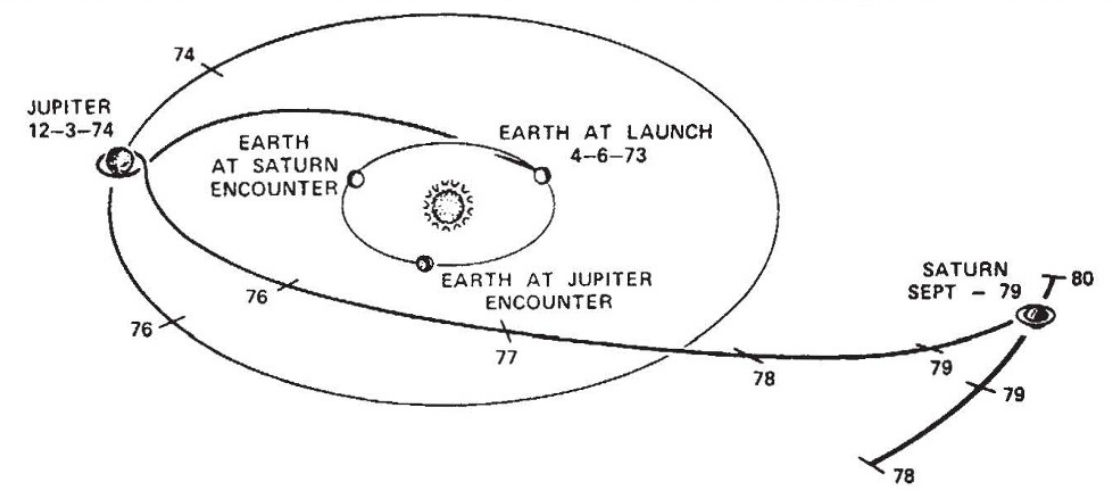

